\title{
Fatores de Risco Cardiovasculares em Adolescentes: Indicadores Biológicos e Comportamentais
}

\author{
Cardiovascular Risk Factors in Adolescents: Biological and Behavioral Indicators
}

\begin{abstract}
Dartagnan Pinto Guedes, Joana Elisabete Ribeiro Pinto Guedes, Decio Sabbatini Barbosa, Jair Aparecido de Oliveira, Luiz Cláudio Reeberg Stanganelli

Universidade Estadual de Londrina - Londrina, PR
\end{abstract}

\section{Objetivo}

Oferecer informações descritivas e investigar a extensão com que os fatores de risco para doenças cardiovasculares de natureza comportamental estão associados aos fatores de risco biológicos na população jovem.

\section{Métodos}

Amostra de 452 adolescentes (246 moças e 206 rapazes) com idades entre 15 e 18 anos, selecionados de uma escola de ensino médio da cidade de Londrina, Paraná. Fatores de risco de natureza comportamental foram analisados mediante prática insuficiente de atividade física, ingestão excessiva de gorduras e de colesterol e uso de tabaco. Como indicadores dos fatores de risco biológicos recorreu-se ao sobrepeso, aos níveis elevados de pressão arterial e concentrações não-favoráveis de lipídios-lipoproteínas plasmáticas.

\section{Resultados}

Por volta de $20 \%$ das moças e $16 \%$ dos rapazes apresentaram pelo menos um fator de risco biológico para o desenvolvimento de doenças cardiovasculares. 0 sobrepeso se associou significativamente com a ingestão excessiva de gorduras, enquanto a pressão arterial elevada se relacionou com o estilo de vida sedentário e o uso de tabaco. A excessiva ingestão de gorduras e de colesterol indicou risco aumentado de concentrações indesejáveis de lipídios-lipoproteínas plasmáticas. Adolescentes fumantes tenderam a demonstrar risco de pressão arterial e de lipídioslipoproteínas plasmáticas alterados duas vezes maior que não-fumantes.

\section{Conclusão}

Os resultados reforçam a necessidade de que intervenções direcionadas à adoção de um estilo de vida saudável, incluindo prática regular de atividade física, padrões dietéticos adequados e abstenção ao uso de tabaco, deverão ser iniciadas em idades jovens.

\section{Palavras-chave}

Pressão arterial, lipídios-lipoproteínas plasmáticas,

\section{Objective}

To provide descriptive information and investigate to what extent behavioral risk factors for cardiovascular diseases are associated with biological risk factors in the young population.

\section{Methods}

The sample included 452 adolescents (246 girls and 206 boys) between 15 and 18 years of age selected in a high school in the city of Londrina, Paraná. Risk factors of a behavioral nature were analyzed as regards insufficient physical activity, excessive intake of fat and cholesterol and smoking. As biological risk factor indicators we used overweight, high arterial pressure levels, and adverse concentration of serum lipids and lipoproteins.

\section{RESULTS}

Approximately $20 \%$ of the girls and $16 \%$ of the boys presented at least one biological risk factor for development of cardiovascular diseases. Overweight was significantly associated with excessive intake of fats, while elevated arterial pressure was related to sedentary lifestyle and smoking. Excessive intake of fat and cholesterol indicated increased risk of undesirable concentrations of serum lipids and lipoproteins. The risk associated with altered blood pressure and serum lipids and lipoprotein was increased two-fold in smokers when compared with nonsmokers.

\section{Conclusion}

The results reinforce the need for interventions targeted at the adoption of a healthy lifestyle, including the regular practice of physical exercise, appropriate dietary patterns, and abstention from smoking as of an early age.

\section{KEY WORDS}

Blood pressure, serum lipids-lipoproteins, overweight, physical activity, dietary patterns, smoking. 
Apesar da discreta tendência de declínio nos casos de morte, as disfunções associadas ao sistema cardiovascular constituem uma das principais causas de morbidade e mortalidade nos países industrializados ${ }^{1}$. No Brasil, estima-se que as doenças cardiovasculares (DCV) respondam por aproximadamente $20 \%$ dos óbitos por causas conhecidas em sujeitos a partir dos vinte anos de idade ${ }^{2}$.

A literatura apresenta fortes evidências no sentido de que as DCV, manifestadas na idade adulta, resultam de complexa interação entre uma variedade de fatores de risco que podem ter origem na infância e na adolescência ${ }^{3-5}$. Portanto, aqueles jovens que eventualmente venham a apresentar fatores de risco, com o avanço da idade, tendem a apresentar maior predisposição ao aparecimento de processos aterosclerótico e conseqüente desenvolvimento das DCV.

Em sendo assim, tentar detectar precocemente a presença de fatores de risco no jovem possibilita o planejamento e a implementação de programas intervencionistas preventivos direcionados à redução da probabilidade de manifestação das DCV futuramente na idade adulta.

As características dos fatores de risco predisponentes às DCV são tradicionalmente identificadas como de natureza biológica, como é o caso da quantidade de gordura corporal, do perfil lipídico-lipoprotéico plasmático e dos níveis de pressão arterial. Contudo, em se tratando de sujeitos jovens, momento em que são adquiridos e incorporados comportamentos de difícil modificação em idades futuras, torna-se prudente analisar não apenas os fatores de risco biológicos de forma isolada, mas também aqueles de natureza comportamental, como são os casos da prática de atividade física, dos hábitos alimentares e do uso de tabaco6-8.

Informações disponíveis quanto à incidência e à agregação de fatores de risco predisponentes às DCV de natureza biológica e comportamental em adolescentes são relativamente escassos. Ao consultar a literatura brasileira, verifica-se que estudos com essa finalidade são raros ou mesmo inexistentes. Nesse sentido, o objetivo do presente estudo foi: (a) oferecer informações descritivas quanto aos fatores de risco predisponentes às DCV observadas em amostra representativa de adolescentes; e (b) estabelecer a extensão com que os fatores de risco de natureza comportamental podem estar associados aos fatores de risco de natureza biológica na população jovem.

\section{Métodos}

Para elaboração do estudo foram utilizadas informações contidas no banco de dados construído a partir do projeto de pesquisa "Atividade Física, Composição da Dieta e Fatores de Risco Predisponentes às Doenças Cardiovasculares em Adolescentes", que inclui adolescentes entre quinze e dezoito anos de idade de ambos os sexos ${ }^{9}$.
0 projeto de pesquisa teve como alvo escolares regularmente matriculados no ensino médio do Colégio de Aplicação ligado à Universidade Estadual de Londrina, Paraná. Optou-se por envolver sujeitos que freqüentavam unicamente essa escola, por conta das características longitudinais do estudo (experimentação de programas de educação para a saúde mediante intervenções dietéticas e de prática de exercícios físicos), e por sua representatividade no universo de escolares de ensino médio do município de Londrina, Paraná.

Protocolos de intervenção no estudo foram aprovados pelo Comitê de Ética em Pesquisa da Universidade Estadual de Londrina e acompanham normas da Resolução 196/96 do Conselho Nacional de Saúde sobre pesquisa envolvendo seres humanos. Inclusão dos sujeitos no estudo ocorreu por desejo em participar do experimento e mediante autorização dos pais ou responsáveis. Para tanto, todos os escolares matriculados no ano letivo de 2003, juntamente com seus pais ou responsáveis, foram contatados e informados quanto à natureza e aos objetivos do estudo. Dos 518 escolares matriculados, 452 (87\%) concordaram em participar do estudo (tab. 1).

\begin{tabular}{|c|c|c|c|}
\hline \multicolumn{4}{|c|}{$\begin{array}{c}\text { Tabela } 1 \text { - Número de sujeitos analisados no } \\
\text { projeto "Atividade Física, Composição da Dieta } \\
\text { e Fatores de Risco Predisponentes às Doenças } \\
\text { Cardiovasculares em Adolescentes" }\end{array}$} \\
\hline Idade & Moças & Rapazes & Total \\
\hline 15 Anos & 52 & 42 & 94 \\
\hline 16 Anos & 75 & 61 & 136 \\
\hline 17 Anos & 64 & 56 & 120 \\
\hline 18 Anos & 55 & 47 & 102 \\
\hline $15-18$ Anos & 246 & 206 & 452 \\
\hline
\end{tabular}

Como informação adicional da amostra analisada no estudo, destaca-se que, com base nos critérios de classificação socioeconômica das famílias dos escolares, mediante informações quanto ao nível de escolaridade do chefe da família, às condições de moradia, à posse de utensílios domésticos, automóveis e número de empregados domésticos ${ }^{10}$, observou-se que $22 \%$ dos escolares foram categorizados em menor nível socioeconômico, 27\% em maior nível e 51\% em níveis intermediários. Com relação aos critérios de classificação de maturação sexual sugeridos por Tanner ${ }^{11}$, 10\% das moças analisadas apresentavam desenvolvimento mamário equivalente ao estágio III, $61 \%$ ao estágio IV e $29 \%$ ao estágio V. Entre os rapazes, $49 \%$ se encontravam no estágio IV de desenvolvimento de pilosidade pubiana, e os $51 \%$ restantes no estágio V.

Os fatores de risco predisponentes às doenças cardiovasculares foram analisados mediante indicadores biológicos e comportamentais. Com referência aos indicadores biológicos, recorreu-se as informações voltadas ao índice de massa corporal, à pressão arterial em repouso e ao teor sangüíneo de lípides-lipoproteínas circulantes. No 
campo comportamental foram obtidas informações acerca do nível de prática da atividade física, da composição da dieta e do uso de tabaco.

O índice de massa corporal foi calculado considerandose a razão entre o peso corporal e o quadrado da estatura $\left(\mathrm{kg} / \mathrm{m}^{2}\right)$. Para determinação da estatura utilizou-se estadiômetro com escala de medida de $0,1 \mathrm{~cm}$, e para a verificação do peso corporal balança antropométrica com definição de 100 g, a partir da metodologia apresentada por Gordon e cols. ${ }^{12}$. Atendendo valores de referência para idade e sexo sugeridos na literatura ${ }^{13}$, foram considerados com sobrepeso aqueles adolescentes que apresentavam IMC $\geq 25 \mathrm{~kg} / \mathrm{m}^{2}$.

Os níveis de pressão arterial sistólica (PAS) e diastólica (PAD) foram aferidos mediante o método auscultatório com auxílio de esfigmomanômetro de coluna de mercúrio, com manguitos de tamanhos apropriados à circunferência dos braços dos adolescentes. Com o adolescente sentado, após um período mínimo de cinco minutos de repouso, a pressão arterial foi medida no braço esquerdo. 0 valor da PAS correspondeu à fase I de Korotkoff e o da $P A D$ à fase $\mathrm{V}$, ou de desaparecimento dos sons. Foram realizadas duas medidas, e o valor médio de ambas foi considerado para efeito de análise. Seguindo os critérios estabelecidos pelo III Consenso Brasileiro de Hipertensão Arterial ${ }^{14}$, foram considerados portadores de pressão arterial elevada aqueles adolescentes que apresentavam PAS $\geq 140 \mathrm{mmHg}$ e PAD $\geq 90 \mathrm{mmHg}$.

As dosagens dos lipídios-lipoproteínas plasmáticas foram realizadas mediante coleta de amostras de $10 \mathrm{ml}$ de sangue venoso na prega do cotovelo, após período de 10-12 horas em jejum, entre 7h00 e 8h00 da manhã. 0 soro foi imediatamente separado por centrifugação, sendo determinados teores de triglicerídeos (TG), colesterol total (CT) e frações, lipoproteínas de baixa densidade (LDL-C) e de alta densidade (HDL-C). Determinou-se o colesterol sérico total pelo método enzimático colesterol oxidase/peroxidase em aparelho espectofotômetro. 0 HDL-C foi medido pelo método reativo precipitante, e o LDL-C calculado pela fórmula de Friedewald e cols. ${ }^{15}$. Os triglicerídeos séricos foram determinados pelo método enzimático glicerol. Os valores de referência empregados para definir um perfil lipídico-lipoproteico de risco aterogênico acompanharam proposta apresentada para adolescentes no Consenso Brasileiro sobre Dislipidemias ${ }^{16}$ : TG $\geq 130 \mathrm{mg} / \mathrm{dl}, \mathrm{CT} \geq 200 \mathrm{mg} / \mathrm{dl}$, LDL-C $\geq 130 \mathrm{mg} / \mathrm{dl} \mathrm{e}$ $\mathrm{HDL}-\mathrm{C} \leq 35 \mathrm{mg} / \mathrm{dl}$.

Para estimar os níveis de prática da atividade física utilizou-se do instrumento retrospectivo de auto-recordação das atividades diárias, preconizado por Bouchard e cols. ${ }^{17}$. Nesse caso, as atividades do cotidiano são classificadas em um continuum envolvendo nove categorias, de acordo com estimativas quanto ao custo calórico médio das atividades realizadas por humanos: (1) repouso na cama; (2) atividades realizadas em posição sentada; (3) atividades leves realizadas em posição de pé; (4) atividades que exigem caminhadas leves ( $<4 \mathrm{~km} / \mathrm{hora}$ ); (5) trabalho manual leve; (6) atividades de lazer ativo e prática de esportes recreativos; (7) trabalho manual em ritmo moderado; (8) atividades de lazer ativo e prática de esportes de intensidade moderada; e (9) trabalho manual intenso e prática de esportes competitivos.

0 instrumento retrospectivo foi preenchido pelos próprios adolescentes em quatro dias da mesma semana, dois no meio (entre segunda-feira e sexta-feira) e dois no final de semana (sábado e domingo). Para efeito de cálculo, utilizou-se média ponderada envolvendo os dois dias do meio e do final de semana. Para sua administração, o dia foi dividido em 96 períodos de quinze minutos cada. Os participantes do estudo receberam instruções e recomendações no sentido de identificar o tipo de atividade, classificada entre as categorias 1 e 9, realizada em cada período de quinze minutos, durante as 24 horas do dia. Lista de atividades características do cotidiano, exemplificando atividades contempladas nas diferentes categorias, foi apresentada aos adolescentes na tentativa de facilitar o preenchimento do instrumento. Ainda, os participantes do estudo foram instruídos a realizar anotações adicionais, caso alguma atividade realizada não estivesse sendo contemplada na lista especificamente elaborada para essa finalidade.

De posse dessas informações, estabeleceu-se o tempo despendido por cada participante do estudo nas diferentes categorias de atividade física. Considerou-se 0 tempo despendido nas categorias 6 a 9 (custo energético médio > 4,8 Mets) como indicador de atividade física de moderada-a-vigorosa intensidades (AFMV). 0 tempo despendido nas categorias 3 a 5 (custo energético médio entre 2,3 e 3,3 Mets) como indicador de atividade física de baixa intensidade (AFBA); e nas categorias 1 e 2 (custo energético < 1,5 Mets) como indicador de inatividade física (INAF). Adicionalmente, com base nas referências calóricas sugeridas pelos idealizadores do instrumento de medida utilizado ${ }^{17}$, recorreu-se às estimativas do dispêndio energético por quilograma de peso corporal das atividades realizadas durante o dia ( $\mathrm{kcal} / \mathrm{kg} / \mathrm{dia}$ ) como indicador da prática habitual de atividade física (DE). Esse procedimento foi validado para utilização em adolescentes diante da técnica critério água duplamente marcada ${ }^{18}$. De acordo com proposta idealizada por $\mathrm{Cale}^{19}$, considerouse como insuficientemente ativos fisicamente aqueles adolescentes que apresentavam DE $\leq 37 \mathrm{kcal} / \mathrm{kg} / \mathrm{dia}$.

As informações acerca da composição da dieta foram obtidas a partir de registros dietéticos. Os participantes do estudo receberam instrumento com instruções e recomendações visando identificar todo alimento ingerido, incluindo bebidas, com suas respectivas porções estimadas (medidas caseiras), em quatro dias da mesma semana, dois no meio (entre segunda-feira e sexta-feira) e dois no final de semana (sábado e domingo). Para efeito de cálculo, utilizou-se a média ponderada envolvendo os dois dias do meio e do final de semana. De posse 
dessas informações, determinou-se a proporção média do suprimento energético/dia proveniente da ingestão das gorduras total $\left(G O R D_{\text {total }}\right)$ e da gordura saturada $\left(A G_{\text {saturado }}\right)$, e a quantidade média/dia quanto à ingestão de colesterol (mg/dia). 0 equivalente calórico e as proporções nutricionais dos alimentos foram estabelecidos mediante utilização do software Sistema de Apoio à Decisão em Nutrição - Versão 2.5, idealizado pelo Centro de Informática em Saúde da Escola Paulista de Medicina/Universidade Federal de São Paulo ${ }^{20}$. A adequação dos indicadores dietéticos foi analisada mediante recomendações da Academia Americana de Pediatria ${ }^{21}$. Considerou-se como adequado consumo $\leq 30 \%$ de gordura total, $\leq 10 \%$ de gordura saturada e $\leq 300 \mathrm{mg} /$ dia de colesterol.

O hábito quanto ao uso de tabaco foi identificado mediante análise das respostas apresentadas pelos adolescentes a uma questão elaborada especificamente para essa finalidade. A questão "você é fumante" foi apresentada aos adolescentes com cinco opções de resposta: (a) não, nunca fumei; (b) não, mas já fumei anteriormente; (c) sim, fumo ocasionalmente; (d) sim, fumo pelo menos uma vez por semana; e (e) sim, fumo diariamente. Foram considerados fumantes aqueles adolescentes que disseram fumar pelo menos uma vez por semana ou diariamente.

O tratamento estatístico das informações foi realizado mediante o pacote computadorizado Statistical Package for the Social Science (SPSS), versão 12.0. Para análise das variáveis contínuas recorreu-se aos procedimentos da estatística descritiva e, posteriormente, para identificação de eventuais diferenças entre os sexos, ao teste "t" de
Student. As variáveis categóricas (proporção de freqüência de acordo com os pontos de corte considerados) foram analisadas mediante tabelas de contingência, envolvendo testes de qui-quadrado $\left(\chi^{2}\right)$ para identificação de diferenças estatísticas entre os sexos. A extensão com que os indicadores relacionados à prática de atividade física, à composição da dieta e ao uso de tabaco possam estar associados ao sobrepeso e aos níveis comprometedores de pressão arterial e de lipídios-lipoproteína plasmática foi estabelecida mediante estimativas de Odds Ratio (OR), por intermédio da utilização da análise de regressão logística binária. Adotou-se intervalo de confiança de 95\%.

\section{Resultados}

Informações estatísticas com relação às variáveis analisadas são mostradas na tabela 2 . No que se refere aos indicadores de natureza biológica associados aos fatores de risco predisponentes às DCV, quando da comparação dos valores médios relativos ao IMC, não se encontram diferenças entre os sexos que possam ser apontadas estatisticamente. No entanto, constatam-se valores médios referentes à PAD e à PAS significativamente mais elevados entre os rapazes. Quanto aos teores plasmáticos de lipídios e de lipoproteínas, os resultados apontam valores médios estatisticamente mais elevados entre as moças na maioria dos componentes analisados. As concentrações médias de TG mostram ser semelhantes entre os sexos.

Com relação aos indicadores de natureza comportamental associados aos fatores de risco predisponentes

\begin{tabular}{|c|c|c|c|c|}
\hline & $\begin{array}{c}\text { Moças } \\
(n=246)\end{array}$ & $\begin{array}{l}\text { Rapazes } \\
(n=206)\end{array}$ & $\begin{array}{l}\text { Teste } \\
\text { "t" }\end{array}$ & $p<t$ \\
\hline \multicolumn{5}{|c|}{ Indicadores biológicos } \\
\hline IMC $\left(\mathrm{kg} / \mathrm{m}^{2}\right)$ & $21,11 \pm 2,81$ & $21,37 \pm 3,17$ & 0,924 & ns \\
\hline PAD (mmHg) & $71,41 \pm 8,61$ & $73,86 \pm 7,91$ & 3,126 & 0,002 \\
\hline PAS (mmHg) & $109,47 \pm 9,56$ & $118,63 \pm 10,20$ & 9,84 & 0 \\
\hline $\mathrm{CT}(\mathrm{mg} / \mathrm{dl})$ & $146,23 \pm 23,36$ & $134,32 \pm 23,21$ & 5,414 & 0 \\
\hline HDL-C (mg/dl) & $52,52 \pm 12,14$ & $46,44 \pm 6,77$ & 6,402 & 0 \\
\hline LDL-C (mg/dl) & $77,70 \pm 22,66$ & $72,14 \pm 20,88$ & 2,692 & 0,008 \\
\hline $\mathrm{TG}(\mathrm{mg} / \mathrm{dl})$ & $80,00 \pm 35,71$ & $81,49 \pm 37,17$ & 0,434 & ns \\
\hline \multicolumn{5}{|c|}{ Atividade física } \\
\hline$D E_{\text {total }}(\mathrm{kcal} / \mathrm{kg} / \mathrm{dia})$ & $36,63 \pm 2,22$ & $37,70 \pm 3,32$ & 4,082 & 0 \\
\hline INAF (min/dia) & $1.199,33 \pm 105,18$ & $1.204,39 \pm 117,56$ & 0,483 & ns \\
\hline AFBA (min/dia) & $233,05 \pm 103,01$ & $199,13 \pm 109,57$ & 3,387 & 0 \\
\hline AFMV (min/dia) & $12,46 \pm 3,48$ & $45,13 \pm 7,21$ & 65,509 & 0 \\
\hline \multicolumn{5}{|c|}{ Indicadores dietéticos } \\
\hline $\mathrm{SE}_{\text {total }}(\mathrm{kcal} / \mathrm{kg} / \mathrm{dia})$ & $34,34 \pm 14,34$ & $39,94 \pm 12,95$ & 4,37 & 0 \\
\hline $\mathrm{GORD}_{\text {total }}(\%)$ & $32,86 \pm 6,92$ & $34,17 \pm 6,98$ & 4,72 & 0 \\
\hline$A G_{\text {saturado }}(\%)$ & $18,18 \pm 2,81$ & $19,61 \pm 3,65$ & 4,702 & 0 \\
\hline Colesterol (mg/dia) & $295,06 \pm 108,95$ & $356,42 \pm 125,08$ & 5,573 & 0 \\
\hline
\end{tabular}


às $D C V$, estimativas quanto ao $D E$, equivalentes às atividades físicas realizadas durante o dia, e os tempos médios despendidos nas atividades físicas de AFBA e de AFMV apontaram diferenças significativas em linguagem estatística entre os sexos. Porém, moças e rapazes permaneceram igualmente por volta de vinte horas/dia inativos fisicamente. Em média, rapazes dedicaram quatro vezes mais tempo do dia em AFMV que moças $(45,13$ versus $12,46 \mathrm{~min} / \mathrm{dia} ; \mathrm{p}=0,000)$. Resultados quanto à composição da dieta também revelam diferenças estatísticas entre os sexos. Os valores médios encontrados apontam os rapazes como tendo apresentado ingestão significativamente maior de gorduras e de colesterol, assim como suplemento energético/dia superior em comparação com as moças.

Proporções de adolescentes que apresentaram indicadores biológicos e comportamentais considerados de risco cardiovascular são mostradas na tabela 3. Os fatores de risco associados aos aspectos comportamentais foram aqueles de maior prevalência. Nesse caso, as moças apresentaram proporção de prática insuficiente de atividade física ( $D E \leq 37 \mathrm{kcal} / \mathrm{kg} / \mathrm{dia}$ ) estatisticamente superior aos rapazes (55,4 versus 41,9\%; $p=0,006)$. As ingestões excessivas de gorduras total e saturada não apresentaram diferenças significativas entre ambos os sexos; porém, se mostraram como os indicadores comportamentais agressivos mais freqüentemente observados. Com diferenças apontadas em linguagem estatística ( $p=0,000$ ), proporção mais elevada de rapazes (50\%) apresentou ingestão $\geq 300 \mathrm{mg} / \mathrm{dia}$ de colesterol dietético em comparação com as moças $(24,8 \%)$. A proporção de fumantes também foi significativamente superior entre os rapazes $(20,7$ versus $15,1 ; p=0,034)$.

Ao recorrer aos valores de referência sugeridos pela Sociedade Brasileira de Cardiologia ${ }^{16}$, verifica-se que as concentrações de lipídios-lipoproteínas plasmáticas associadas ao aumento do risco de DCV foram comuns em aproximadamente $2 \%-13 \%$ das moças estudadas. Entre os rapazes, a proporção de risco oscilou entre $4 \%$ e $8 \%$. Além disso, entre $8 \%$ e $10 \%$ dos adolescentes de ambos os sexos selecionados no estudo demonstraram níveis adversos de pressão arterial, sugerindo que eventual indicação de hipertensão não é fenômeno incomum na adolescência. Diferenças significativas, apontando maior proporção de moças com comprometimento lipoprotéico plasmático, foram identificadas quando das comparações entre ambos os sexos do CT (12,9 versus 5,6\%; $p=$ $0,015)$ e de suas frações HDL-C (1,9 versus $4,4 \%$; $p$ $=0,045)$ e LDL-C (9,0 versus $4,4 \% ; p=0,006)$. As diferenças quanto às proporções de moças $(9,5 \%)$ e de rapazes $(8,1 \%)$ que apresentaram valores elevados de TG não foram apontadas em linguagem estatística.

A tabela 4 descreve as proporções de adolescentes

Tabela 3 - Proporção (\%) de adolescentes que apresentaram fatores de risco predisponentes às doenças cardiovasculares: indicadores biológicos e comportamentais

\begin{tabular}{|c|c|c|c|c|}
\hline & Moças & Rapazes & Teste $\chi^{2}$ & $\mathrm{p}<\mathrm{t}$ \\
\hline \multicolumn{5}{|c|}{ Indicadores biológicos } \\
\hline IMC $\left(\geq 25 \mathrm{~kg} / \mathrm{m}^{2}\right)$ & 16,5 & 18,6 & 0,213 & ns \\
\hline $\operatorname{PAD}(\geq 90 \mathrm{mmHg})$ & 10,2 & 8,1 & 0,366 & ns \\
\hline PAS ( $\geq 140 \mathrm{mmHg}$ ) & 9,7 & 8,8 & 0,027 & ns \\
\hline CT $(\geq 200 \mathrm{mg} / \mathrm{dl})$ & 12,9 & 5,6 & 6,084 & 0,015 \\
\hline HDL-C $(\leq 35 \mathrm{mg} / \mathrm{dl})$ & 1,6 & 4,4 & 2,224 & 0,045 \\
\hline LDL-C ( $\geq 130 \mathrm{mg} / \mathrm{dl})$ & 9 & 4,8 & 7,538 & 0,006 \\
\hline $\mathrm{TG}(\geq 130 \mathrm{mg} / \mathrm{dl})$ & 9,5 & 8,1 & 0,126 & ns \\
\hline \multicolumn{5}{|c|}{ Indicadores comportamentais } \\
\hline $\mathrm{DE}(\leq 37 \mathrm{kcal} / \mathrm{kg} / \mathrm{dia})$ & 55,4 & 41,9 & 7,644 & 0,006 \\
\hline $\operatorname{GORD}_{\text {total }}(\geq 30 \%)$ & 63,7 & 71 & 2,383 & ns \\
\hline$A G_{\text {saturado }}(\geq 10 \%)$ & 57,4 & 62,1 & 0,842 & ns \\
\hline Colesterol ( $\geq 300$ mg/dia) & 24,8 & 50 & 29,716 & 0 \\
\hline Fumante (\%) & 15,1 & 20,7 & 4,152 & 0,034 \\
\hline
\end{tabular}

Tabela 4 - Proporção (\%) de adolescentes que apresentaram fatores de risco predisponentes às doenças cardiovasculares combinados

\begin{tabular}{|c|c|c|c|c|c|c|c|c|c|c|}
\hline \multirow[b]{3}{*}{ Moças } & \multicolumn{5}{|c|}{ Indicadores biológicos } & \multicolumn{5}{|c|}{ Indicadores comportamentais } \\
\hline & Nenhum & 1 & 2 & 3 & $\geq 4$ & Nenhum & 1 & 2 & 3 & $\geq 4$ \\
\hline & 59,1 & 20,4 & 16,3 & 2,7 & 1,5 & 9,5 & 20,9 & 33,5 & 25,9 & 10,1 \\
\hline Rapazes & 68,5 & 16 & 11,6 & 2,9 & 1 & 10,5 & 18,5 & 26,6 & 23,4 & 21 \\
\hline Teste $\chi^{2}$ & 3,873 & 1,168 & 1,669 & 0,025 & 0,003 & 0,038 & 0,27 & 2,209 & 0,254 & 9,574 \\
\hline $\mathrm{p}<\mathrm{t}$ & 0,049 & ns & ns & ns & ns & ns & ns & ns & ns & 0,003 \\
\hline
\end{tabular}


analisados que apresentaram múltiplos fatores de riscos concomitantemente. Por volta de $20 \%$ das moças e $16 \%$ dos rapazes demonstraram ser portadores de pelo menos um fator de risco biológico relacionado ao desenvolvimento de DCV. Concomitâncias de dois e três potenciais fatores de risco biológico foram identificadas em $16 \%$ e $3 \%$ das moças e $12 \%$ e $3 \%$ dos rapazes, respectivamente. Diferenças significativas entre ambos os sexos foram constatadas somente quanto a menor proporção de moças que não apresentou pelo menos um fator de risco biológico (59,1 versus $68,5 \%$; $p=$ $0,049)$. Quanto aos fatores de risco relacionados aos indicadores comportamentais, cerca de tão-somente 10\% dos adolescentes deixaram de apresentar pelo menos um dos cinco comportamentos agressivos selecionados no estudo que possam eventualmente predispor ao desenvolvimento de DCV. As moças apresentaram proporções de magnitudes crescentes até a presença combinada de três fatores de risco comportamentais, enquanto os rapazes demonstraram distribuição equilibrada nas proporções de ocorrência simultânea dos cinco fatores de risco de natureza comportamental considerados. Diferenças estatísticas, apontando maiores proporções entre os rapazes, foram identificadas quanto à presença simultânea de quatro ou mais fatores de risco comportamentais (21 versus 10,1\%; $p=0,003$ ).

A extensão com que os fatores de risco de natureza comportamental (atividade física insuficiente, ingestão excessiva de gorduras e de colesterol e uso de tabaco) se associaram aos fatores de risco de natureza biológica (sobrepeso, níveis comprometedores de pressão arterial e de lipídios-lipoproteínas plasmáticas) foi estimada mediante análise de regressão logística binária. Dimensões de OR provenientes dos cálculos estatísticos são apresentadas na tabela 5. Os resultados encontrados indicam que a ingestão excessiva de gordura total e de gordura saturada foram os indicadores comportamentais que apresentaram mais elevada associação com a ocorrência do sobrepeso entre os adolescentes de ambos os sexos analisados no estudo. Baseando-se nos valores de OR, estima-se que, em linguagem estatística, moças que demonstraram ingestão excessiva de gordura total (OR $=1,64 ; 95 \%$ IC 1,32-1,98) e de gordura saturada $(\mathrm{OR}=1,57 ; 95 \%$ IC 1,28-1,88) apresentaram, respectivamente, $64 \%$ e $57 \%$ mais chances de serem portadores de sobrepeso. No caso dos rapazes, aqueles que demonstraram ingestão excessiva de gorduras total (OR $=2,17 ; 95 \%$ IC 1,81-2,58) e de gordura saturada $(\mathrm{OR}=1,93 ; 95 \%$ IC 1,65-2,24) apresentaram, em valores aproximados, duas vezes mais chances de ser portadores de sobrepeso. Ingestão excessiva de colesterol, uso de tabaco e prática insuficiente de atividade física não apresentaram associações significativas com a ocorrência do sobrepeso.

Prática insuficiente de atividade física e uso de tabaco foram os indicadores comportamentais que apresentaram mais elevada associação com níveis comprometedores de pressão arterial, sobretudo no caso da PAS. Ingestão

\section{Tabela 5 - Odds Ratio entre indicadores biológicos e comportamentais associados aos fatores de risco} predisponentes às doenças cardiovasculares de adolescentes

Atividade física Insuficiente

IMC

Moças $\quad 1,17(0,94-1,41)$

Rapazes

$1,06(0,83-1,31)$

LDL-C Moças
Rapazes

TG Moças

Moças $\quad 1,80(1,63-2,01)$

Rapazes

$$
1,89(1,50-2,16)
$$

$1,14(0,96-1,29)$

$1,10(0,90-1,28)$

$1,91(1,66-2,16)$

$1,62(1,31-1,89)$

$1,27(1,02-1,48)$

$1,90(1,64-2,12)$

$1,93(1,66-2,17)$

$1,61(1,42-1,83)$

$0,92(0,73-1,13)$

$0,62(0,51-0,70)$

$0,48(0,39-0,56)$

$1,13(0,87-1,35)$

$0,96(0,76-1,18)$

$0,87(0,64-1,08)$

$0,63(0,44-0,85)$

$1,82(1,55-2,05)$

$1,49(1,31-1,69)$

$1,10(0,88-1,29)$

$1,88(1,68-2,12)$

$1,60(1,43-1,81)$
Ingestão excessiva

Gordura saturada

$1,57(1,28-1,88)$

$1,93(1,65-2,24)$

$1,17(0,94-1,36)$

$1,41(1,07-1,77)$

$1,24(1,05-1,41)$

$1,74(1,51-1,99)$

$2,42(2,04-2,81)$

$1,91(1,61-2,25)$

$0,74(0,59-0,88)$

$0,44(0,31-0,55)$

$2,52(2,15-2,80)$

$1,61(1,30-1,88)$

$2,64(2,27-2,99)$

$1,78(1,52-2,04)$

\section{Colesterol}

$1,19(0,98-1,37)$

$1,21(0,99-1,37)$

$1,11(0,90-1,30)$

$0,84(0,69-0,98)$

$1,36(1,19-1,53)$

$1,15(0,94-1,32)$

$1,72(1,49-1,98)$

$1,50(1,26-1,71)$

$0,66(0,49-0,80)$

$0,56(0,40-0,70)$

$1,51(1,30-1,74)$

$1,23(1,01-1,48)$

$2,47(2,15-2,83)$

$1,61(1,39-1,86)$
Uso de

Tabaco

$0,86(0,71-0,99)$

$0,91(0,69-1,11)$

$1,86(1,56-2,14)$

$1,99(1,65-2,31)$

$2,27(1,85-2,71)$

$2,41(2,01-2,85)$

$2,33(1,98-2,70)$

$1,79(1,40-2,15)$

$2,27(1,85-2,71)$

$1,91(1,46-2,34)$

$2,79(2,40-3,20)$

$2,65(2,34-2,98)$

$2,65(2,25-3,02)$

$2,56(2,17-2,99)$

IMC - índice de massa corpórea; PAD - pressão arterial diastólica; PAS - pressão arterial sistólica; CT - colesterol total e TG - triglicérides 
excessiva de gorduras e de colesterol apresentou menores associações com eventual elevação dos níveis de pressão arterial, apesar de que, no caso específico da PAS, os valores de OR demonstraram significância estatística. Moças (OR $=2,27 ; 95 \%$ IC 1,85-2,71) e rapazes (OR $=2,41 ; 95 \%$ IC 2,01-2,85) fumantes apresentaram predisposição superior a duas vezes mais para elevação da PAS que seus pares não-fumantes. Quanto à prática de atividade física, moças (OR $=1,91 ; 95 \%$ IC 1,66$21,16)$ e rapazes (OR $=1,90 ; 95 \%$ IC 1,64-2,12) insuficientemente ativos fisicamente demonstraram por volta de $90 \%$ mais chances de apresentarem valores de PAS $\geq 140 \mathrm{mmHg}$.

Com relação aos lipídios-lipoproteínas, dimensões de OR encontradas revelam que o uso de tabaco e a ingestão excessiva de gordura saturada foram os indicadores comportamentais que mais contribuíram para a preocupante alteração de seus níveis plasmáticos. Aquelas moças fumantes ou que fazem uso de excessiva ingestão de gordura saturada demonstram possuir de $2,3(\mathrm{CT}-\mathrm{OR}=2,33 ; 95 \%$ IC $1,98-2,70)$ a 2,8 (LDL$\mathrm{C}-\mathrm{OR}=2,79 ; 95 \%$ IC 2,40-3,20) mais chances de apresentarem perfil aterogênico de lipídios-lipoproteínas plasmáticas. No caso dos rapazes, as dimensões encontradas de OR se reduziram discretamente; contudo, ainda assim, permaneceram significativos em linguagem estatística. De acordo com o delineamento estabelecido para a regressão logística, as menores dimensões de OR foram encontradas entre os valores de LDL-C $\geq 130$ $\mathrm{mg} / \mathrm{dl}$ e a prática insuficiente de atividade física e entre os valores de HDL-C $\leq 35 \mathrm{mg} / \mathrm{dl}$ e a ingestão excessiva de gorduras e de colesterol. Porém, concentrações diminuídas de HDL-C apresentaram associações significativas com o uso de tabaco (moças - OR = 2,27; 95\% IC 1,85-2,71; e rapazes $-\mathrm{OR}=1,91 ; 95 \% \mathrm{IC} 1,46-2,34)$.

\section{Discussão}

Estudos prévios disponibilizados na literatura procuram descrever estimativas quanto à prevalência de fatores de risco predisponentes às DCV em populações jovens. No entanto, enquanto alguns desses estudos têm enfocado unicamente indicadores de natureza biológica ${ }^{22-28}$; outros concentram suas informações tão-somente em indicadores comportamentais agressivos à saúde cardiovascular ${ }^{29-31}$. O presente estudo, além de oferecer informações descritivas conjuntas de natureza biológica e comportamental observadas em amostra de adolescentes, tentou estabelecer a extensão com que selecionados indicadores comportamentais possam estar associados aos indicadores biológicos considerados de risco para 0 aparecimento e o desenvolvimento das DCV.

Com relação aos fatores de risco biológicos predisponentes às DCV, diferenças entre ambos os sexos quanto aos valores médios de IMC, pressão arterial e concentrações plasmáticas de lipídios-lipoproteínas observados no estudo coincidem com tendências encontradas na literatura. Indicações encontradas em publicações anteriores apontam que o dimorfismo sexual observado nos níveis de pressão arterial e de lipídio-lipoproteínas plasmáticas pode ser explicado em razão das alterações hormonais típicas de cada sexo que ocorrem em idades próximas da puberdade. A maior produção de estrogênio que ocorre no organismo feminino nesse período deverá contribuir temporariamente para uma mais elevada concentração plasmática de CT e de suas frações; ao passo que a maior produção de testosterona no organismo masculino está relacionada aos níveis mais elevados de pressão arterial ${ }^{32,33}$. Porém, no início da idade adulta já está estabelecido o padrão lipoprotéico responsável pela maior incidência de DCV entre os homens frente às mulheres. A literatura consultada sugere que eventual relação entre níveis de TG e sexo não é precisa. Alguns estudos encontraram valores mais elevados entre os rapazes ${ }^{27,34-36}$; enquanto outros, coincidindo com os resultados observados no presente estudo, não encontraram diferenças que possam ser apontadas estatisticamente entre os adolescentes de ambos os sexos $23-26,28$.

Em comparação com outros estudos, se, por um lado, os adolescentes aqui analisados apresentaram valores médios quanto aos fatores de risco biológicos predisponentes às DCV similares a de outros estudos realizados em países da América Latina22,26,37, da África ${ }^{23}$ e da Ásia ${ }^{38,39}$; por outro, são constatadas diferenças importantes quando da comparação com resultados provenientes de casuísticas de mesma idade de países europeus ${ }^{24,27,28,33,36,40}$ e norte-americanos ${ }^{25,35}$. Apesar de localizados alguns estudos envolvendo jovens brasileiros ${ }^{41-44}$, diferenças relacionadas à composição das amostras impedem eventuais comparações com os resultados observados no presente estudo. Acreditase que as diferenças quanto aos atributos biológicos relacionados aos fatores de risco predisponentes às DCV possam ser atribuídas, em grande parte, às múltiplas contingências mesológicas definidas pelas características geoclimáticas, econômicas, sociais e culturais, que modulam a expressão do potencial genético.

Quanto à proporção de adolescentes que apresentaram informações de natureza biológica indicativa de risco para as DCV, no que se refere especificamente ao IMC, verificou-se que, em comparação com resultados de levantamentos epidemiológicos envolvendo diferentes segmentos da população de jovens brasileiros ${ }^{45,46}$, menor proporção de adolescentes reunidos no estudo foi classificada com sobrepeso (16,5\% de moças e $18,6 \%$ de rapazes). No entanto, mesmo assim, esses dados devem causar preocupação, considerando que o excesso de peso corporal presente na infância e na adolescência tende a persistir em idades futuras. Estimativas sugerem que, de cada cinco adolescentes com sobrepeso, apenas um deles tende a reduzir e a manter seu peso corporal em limites esperados quando adulto ${ }^{47}$. Também, programas de redução e controle do peso corporal na adolescência 
parecem apresentar eficiência bastante limitada, supondo que aqueles adolescentes com sobrepeso deverão, provavelmente, continuar a apresentar sobrepeso na vida adulta.

Nesse particular, deve-se destacar que o sobrepeso não é considerado um fator de risco biológico imediato para o aparecimento e o desenvolvimento de DCV. Contudo, em geral, perfil cardiovascular comprometido na idade adulta é acompanhado por excesso de peso corporal instalado e mantido quando criança e adolescente ${ }^{48,49}$. Assim, mediante ações de controle do peso corporal em idades jovens, pode-se potencialmente minimizar a ocorrência de outros fatores de risco notórios em idades mais avançadas.

Quanto aos valores de pressão arterial, torna-se lícito salientar que níveis mais elevados, identificados em um a cada dez dos adolescentes analisados, não deverão inferir, necessariamente, a presença de hipertensão. Nesse caso, para conferir a hipertensão, recomenda-se que valores anormalmente elevados de pressão arterial devam ser confirmados em dois ou mais diferentes momentos em determinado período de tempo ${ }^{50}$. Porém, acompanhamentos longitudinais têm demonstrado que, aqueles adolescentes com elevados níveis de pressão arterial tendem a manter os altos valores pressóricos e a serem diagnosticados como portadores de hipertensão mais tarde na idade adulta ${ }^{51}$. Estudos ecocardiográficos em jovens mostram aumento contínuo da espessura da parede ventricular posterior esquerda em paralelo aos níveis crescentes de pressão arterial ${ }^{52}$. Portanto, níveis alterados de pressão arterial na adolescência são demonstrativos de que possa haver necessidade de atendimento terapêutico imediato visando à prevenção da hipertensão futura.

Comparações com resultados observados em outros estudos quanto à proporção de adolescentes que apresentaram comprometimento nos níveis de pressão arterial e de lipídios-lipoproteínas plasmáticas devem ser realizadas com alguma cautela, em razão de eventuais diferenças associadas aos pontos-de-corte considerados. Contudo, com relação aos valores de pressão arterial, torna-se interessante destacar que os adolescentes aqui analisados apresentaram proporções mais favoráveis que adolescentes analisados em estudos europeus ${ }^{24,36}$ e norte-americanos ${ }^{25,35}$; mas com proporções similares aos estudos realizados em países da América Latina ${ }^{37}$, da Ásia ${ }^{38,39}$, da África ${ }^{23}$ e no Brasil ${ }^{53,54}$.

No que se refere às concentrações de lipídioslipoproteínas plasmáticas, consistente com resultados de outros estudos ${ }^{22-28}$, as moças apresentaram proporção de comprometimento significativamente mais elevada nas dosagens de CT e da fração LDL-C. Níveis diminuídos da fração HDL-C, menos freqüentes em ambos os sexos, o que parece ser típico da população jovem, foi o único fator de risco biológico em que os rapazes apresentaram proporção significantemente superior às moças. Esses achados repercutem com grande significado, tendo presente dados de necropsias realizadas em jovens que vieram a óbito no transcurso de estudos prospectivos. Neles foi possível verificar que o perfil desfavorável de lipídios-lipoproteínas plasmáticas no organismo jovem pode ser responsável por lesões anatomopatológicas das artérias coronárias e da aorta, se definindo, portanto, como forte preditor de DCV em idades adultas. A presença de estrias gordurosas e de placas fibrosas esteve relacionada principalmente aos níveis alterados da fração HDL-C e do TG55,56. Em sendo assim, o controle dos níveis plasmáticos dos lipídios-lipoproteínas deverá minimizar a progressão de eventual processo aterosclerótico em adolescentes e, desse modo, reduzir o risco futuro da ocorrência de DCV.

Importante destacar também que, no presente estudo, proporção significativa dos adolescentes analisados (40,9\% das moças e $31,5 \%$ dos rapazes) exibiram pelo menos um fator de risco biológico predisponente às DCV, e aproximadamente $50 \%$ desses adolescentes apresentaram agregação de múltiplos fatores de risco. Sobre essas circunstâncias, e considerando evidências no sentido de que fatores de risco biológicos identificados na adolescência tendem a permanecer na idade adulta ${ }^{57}$, deve-se levar em conta que, quanto maior a quantidade de fatores de risco agregados maior é a probabilidade de aparecimento e desenvolvimento precoce das DCV. Em sendo assim, aqueles adolescentes reunidos no estudo que apresentaram indicadores biológicos isolados ou concomitantes acima dos pontos-de-corte tidos como de risco deverão ser considerados eventuais candidatos a apresentar futura DCV. Somente mediante acompanhamentos longitudinais daqueles adolescentes considerados de risco se poderá confirmar a presença futura dessa patologia. Contudo, mesmo que apenas uma parcela desses adolescentes venha a confirmar esse prognóstico, isto, por si só, já se constitui em número significativo de adolescentes com fator preditivo para as DCV identificado precocemente.

Quanto aos indicadores de natureza comportamental relacionados ao aparecimento e ao desenvolvimento de DCV, verifica-se que os resultados encontrados coincidem com tendências apresentadas por outros estudos, apontando os rapazes como sendo habitualmente mais ativos; porém, com prática dietética vinculada a um maior grau de comprometimento que as moças ${ }^{30,31}$. Embora diferenças metodológicas relacionadas aos instrumentos de medidas empregados na coleta dos dados e aos critérios de interpretação das informações possam comprometer comparações com outros estudos, os resultados apresentados pelos adolescentes aqui analisados apontam uma mais elevada proporção de sedentarismo e de uso de dieta aterogênica.

No que concerne ao uso de tabaco, no presente estudo o ato de fumar pelo menos uma vez por semana ou diariamente foi aceito para definir aqueles 
adolescentes fumantes. Mediante essa definição, a proporção de rapazes que se reportaram como fumantes foi significativamente superior à de moças. Em comparação com estudos disponibilizados na literatura que apresentam características epidemiológicas, esses achados mostram maior proporção de rapazes adolescentes fumantes. Levantamentos realizados em outras regiões brasileiras ${ }^{58,59}$, em países industrializados ${ }^{29-}$ ${ }^{31}$ e em vias de desenvolvimento ${ }^{37,60}$ revelam prevalências similares em ambos os sexos próximas de $15 \%$.

Chamou atenção ainda o fato de que tão-somente $9,5 \%$ das moças e $10,5 \%$ dos rapazes não apresentaram pelo menos um indicador comportamental comprometido. Esta elevada agregação de comportamentos de risco foi também observada em outros estudos ${ }^{61}$, sugerindo que proporção acentuada de adolescentes que apresenta estilo de vida sedentário tende a adotar simultaneamente dieta não-saudável e a fazer uso de tabaco. Esses achados se revestem de enorme importância para a adoção de hábitos saudáveis ao longo de toda a vida, em razão de evidências disponibilizadas na literatura sugerirem que comportamentos não-saudáveis adquiridos e cultivados na infância e na adolescência serem de difícil modificação e tenderem a permanecer nas idades futuras ${ }^{62,63}$.

Análise quanto à extensão com que os comportamentos de risco possam estar associados aos fatores de risco biológicos predisponentes às DCV em populações jovens deverá considerar a participação dos componentes genéticos na variação do peso corporal e dos níveis de pressão arterial e de lipídios-lipoproteínas plasmáticas ${ }^{64}$. Assumindo essa condição, torna-se importante referir que, nesses casos, as eventuais alterações observadas nos fatores de risco biológicos em conseqüência dos comportamentos de risco podem apresentar variações individuais para se manifestarem; além do que, em tese, supõe-se que sua expressão é menos provável de ocorrer em adolescentes que em adultos.

De qualquer forma, mesmo diante dessas limitações, os valores de OR encontrados apontaram associações entre os indicadores comportamentais e a maioria dos fatores de risco biológicos predisponentes às DCV selecionados no estudo. Os resultados encontrados sugerem que a prática insuficiente de atividade física, representada no estudo por $\mathrm{DE} \leq 37 \mathrm{kcal} / \mathrm{kg} / \mathrm{dia}$, em ambos os sexos, pode desempenhar papel mais importante para explicar níveis aumentados de pressão arterial. Aqueles adolescentes menos ativos fisicamente demonstraram de $80 \%$ a $90 \%$ mais chances de apresentarem comprometimentos vinculados aos níveis de pressão arterial do que seus pares mais ativos. Entretanto, consistente com resultados de outros estudos ${ }^{35,36,39,65}$, na presente casuística, concentrações de lipídios-lipoproteínas plasmáticas consideradas de risco e valores mais elevados de peso corporal (IMC $\geq 25 \mathrm{~kg} / \mathrm{m}^{2}$ ) não demonstraram receber influência significativa da prática insuficiente de atividade física.

Em estudos envolvendo adultos, comprova-se que um estilo de vida sedentário é um comportamento claramente identificado com o aparecimento e o desenvolvimento de DCV e de seus fatores de risco associados ${ }^{66}$. Contudo, parece que essa associação não se confirma com o mesmo potencial em populações jovens. No campo especulativo, apresenta-se a hipótese de que, em parte, essas diferenças possam ocorrer em razão das adaptações metabólicas relacionadas ao controle das concentrações dos lipídios-lipoproteínas plasmáticas e do peso corporal, induzidas pela prática habitual de atividade física, de maneira particular no organismo jovem, ainda não totalmente maturado biologicamente, e no adulto. Por exemplo, em tese, assume-se que os adolescentes possam ser mais ativos fisicamente em seu cotidiano e, por esse motivo, as variações dos teores plasmáticos e do peso corporal não apresentam a mesma sensibilidade que em adultos. Também, pode ser que as associações significativas observadas em adultos sejam não-causal, havendo, portanto, efeitos adicionais de outras variáveis não-conhecidas afetando as relações atividade físicalipídios-lipoproteínas plasmáticas e atividade físicapeso corporal, o que ainda não é aparente entre os adolescentes. Isso talvez venha sugerir a concepção de que as repercussões do sedentarismo nas concentrações de lipídios-lipoproteínas plasmáticas e no peso corporal possam levar algum tempo para ser identificadas.

Prática dietética de maior ingestão de gorduras e de colesterol repercutiu significativamente no risco dos adolescentes analisados apresentarem excesso de peso corporal, valores aumentados de pressão arterial e perfil lipídico-lipoprotéico desfavorável. Os valores de OR encontrados comprovam que a extensão das associações entre a prática dietética aterogênica e os fatores de risco biológicos selecionados no estudo apresentaram magnitudes diferentes em cada sexo. Enquanto entre os rapazes a excessiva ingestão de gorduras total e saturada se associou mais intensamente com a presença do sobrepeso, entre as moças proporções inadequadas de gorduras e de colesterol na composição da dieta desempenharam papel mais importante nas maiores concentrações de lipídios-lipoproteínas plasmáticas. No entanto, provavelmente em razão da estreita relação metabólica existente entre os componentes lipídicoslipoprotéico, verificou-se que as dimensões das OR observadas nas associações com a prática dietética aterogênica, em cada sexo, foram bastante similares. Esses achados coincidem com resultados de estudos de intervenção que, ao reduzir a ingestão de gorduras saturadas na prática dietética de jovens, comprovaram diminuição mais acentuada nas concentrações de LDL-C e TG entre as moças ${ }^{67}$.

As menores dimensões de OR observadas entre elevado consumo de colesterol dietético e os fatores de risco biológicos selecionados são consistentes com as fracas associações encontradas em estudos envolvendo adultos ${ }^{68}$, e confirmam evidências no sentido de que a ingestão excessiva de colesterol dietético 
apresenta associação de menor extensão com maiores concentrações de lipídios-lipoproteínas plasmáticas que a ingestão excessiva de gorduras total e saturada.

Ao analisar a extensão da associação entre o uso de tabaco e valores aumentados de pressão arterial e perfil desfavorável de lipídios-lipoproteínas, verificou-se que as dimensões das OR foram, na maioria das situações, superiores a duas unidades. Em sendo assim, na presente casuística, ser um adolescente fumante implica num aumento de no mínimo duas vezes mais na probabilidade de se encontrar valores de pressão arterial e de lipídioslipoproteínas plasmáticas sugestivo de hipertensão e de dislipidemia, respectivamente. Contudo, quando da associação entre o uso de tabaco e a presença do sobrepeso, verificou-se que os valores de OR não foram relevantes em linguagem estatística, sugerindo que aqueles adolescentes fumantes não necessariamente elevam o risco de acumular maior quantidade de peso corporal.

A despeito das indiscutíveis ações deletérias dos componentes do tabaco para a saúde em geral, à semelhança do que foi encontrado em outros estudos ${ }^{69,70}$, evidências reunidas no estudo apontam forte influência do uso de tabaco na presença de fatores de risco biológicos predisponentes às DCV em adolescentes. Efeitos reversíveis associados à saúde cardiovascular em conseqüência do abandono do uso de tabaco têm sido documentados experimentalmente em adultos ${ }^{71}$. Nesses casos, os benefícios alcançados são parcialmente explicados pelas alterações favoráveis observadas nos níveis de pressão arterial e de lipídios-lipoproteínas plasmáticas. A incógnita está em identificar o impacto do abandono do uso de tabaco na saúde cardiovascular de adolescentes.

Considerando a demonstração de sua elevada associação com as agressões aterogênicas e a potencial predisposição presente em idades jovens com relação à aquisição de dependência quanto ao uso de tabaco, torna-se sensato assumir que o risco dos adolescentes fumantes em apresentar posterior DCV na idade adulta é extremamente elevado.

A concepção de que potenciais fatores de risco biológicos possam se instalar precocemente no organismo jovem e avançar por vários estágios de evolução para resultar em DCV na idade adulta, e a confirmação dos achados de que os fatores de risco biológicos podem ser afetados igualmente na adolescência e na idade adulta em resposta à exposição de comportamentos de risco acentuam a importância de idealizar ações profiláticas direcionadas à prevenção e à modificação de hábitos agressivos à saúde cardiovascular dos adolescentes. Nesse particular, embora o contexto sociofamiliar possa exercer forte participação no desenvolvimento de comportamentos que influenciam o modo de vida saudável dos jovens, programas de escolarização direcionados à educação para a saúde deverão ser colocados em prática, na tentativa de auxiliar os jovens a minimizarem o excepcional impacto da mídia quanto à opção por adotarem hábitos de atividades sedentárias voltadas ao lazer e à ocupação do tempo livre, ao maior consumo de alimentos aterogênicos e ao uso de tabaco. Especialistas da área são unânimes em sugerir que, esforços para prevenir e reduzir os comportamentos de risco deverão ser instituídos no período de escolarização, momento em que os jovens estão especialmente receptivos a incorporar em seu cotidiano e a projetar para as idades futuras comportamentos agressivos à saúde.

Nesse contexto, destaca-se que, tão-somente a simples restrição ao acesso de práticas dietéticas inadequadas e ao uso de tabaco e de outras substâncias nocivas, e a prática compulsória de atividade física mediante as aulas de educação física tornam-se pouco prováveis que possam levar os jovens a assumir hábitos saudáveis para toda a vida. Os programas de educação para a saúde deverão contemplar estratégias pedagógicas para auxiliar os jovens a se conscientizarem da nociva influência dos comportamentos de risco e a tomarem suas próprias decisões com relação a adotarem hábitos saudáveis permanentes.

\section{Potencial Conflito de Interesses}

Declaro não haver conflitos de interesses pertinentes.
1. Porkka KVL, Raitakari OT, Leino A, et al. Trends in serum lipid levels during 1980-1992 in children and young adults. Am J Epidemiol. 1997; 146: 64-77.

2. Lessa I. Introdução à epidemiologia das doenças cardiovasculares no Brasil. In: Lessa I. O Adulto Brasileiro e as Doenças da Modernidade. Epidemiologia das Doenças Crônicas Não-Transmissíveis. São Paulo: Editora Hucitec; 1998: 73-6.

3. Berenson GS, Srinivasan SR, Bao W, et al. Association between multiple cardiovascular risk factors and atherosclerosis in children and young adults. N Engl J Med. 1998; 338: 1650-6.

4. Myers L, Coughlin SS, Webber LS, et al. Prediction of adult cardiovascular multifactorial risk status from childhood risk factor levels. Am J Epidemiol. 1995; 142: 918-24.

5. Strong JP, Malcom GT, McMahan CA, et al. Prevalence and extent of atherosclerosis in adolescents and young adults. Implications for prevention from the pathobiological determinants of atherosclerosis in youth study. JAMA. 1999; 281: 727-35.

6. Gidding SS. Preventive pediatric cardiology: tobacco, cholesterol, obesity, and physical activity. Pediatr Clin North Am. 1999; 46: 253-62.

7. Thomas NE, Backer JS, Davies B. Established and recently identified coronary heart disease risk factors in young people. Sports Med. 2003; 33: $633-50$.

8. Twisk J, Boreham C, Cran G, et al. Clustering of biological risk factors for cardiovascular disease and the longitudinal relationship with lifestyle of an adolescent population: The Northern Ireland Young Hearts Project. J Card Risk. 1999; 6: 355-62.

9. Guedes DP, Guedes JERP, Barbosa DS, Oliveira JA. Cardiovascular risk factors in brazilian adolescents. FIEP Bulletin. 2005; 75: 403-9.

10. ANEP. Critério de Classificação Econômica Brasil. São Paulo: Associação Nacional de Empresas de Pesquisa. 2000. 
11. Tanner JM. Growth at Adolescence. $2^{\text {a }}$ ed. Oxford: Blackwell Scientific Publications; 1962

12. Gordon CC, Chumlea WC, Roche AP, et al. Stature, recumbent length, and weight. In: Lohman TG; Roche AF; Martorell R, ed. Anthropometric Standardization Reference Manual. Champaign, Illinois: Human Kinetics Books; 1988: 3-8.

13. Cole TJ, Bellizzi MC, Flegal KM, Dietz WH. Establishing a standard definition for child overweight and obesity worldwide: international survey. BMJ 2000; 320: 1240-3.

14. Consenso Brasileiro de Hipertensão Arterial. III CBH. Rev Bras Clin Ter. 1998; 24: 231-72.

15. Friedewald WT, Levy RI, Frederickson DS. Estimation of the concentration of low density lipoprotein cholesterol in plasma without use of the preparative ultracentrifuge. Clin Chem. 1972; 18: 499502 .

16. Sociedade Brasileira de Cardiologia. Consenso brasileiro sobre dislipidemias: detecção, avaliação e tratamento. Arq Bras Cardiol. 1996; 67: 113-28.

17. Bouchard C, Tremblay A, Le Blanc C, et al. A method to assess energy expenditure in children and adults. Am J Clin Nutr. 1983; 37: 4617.

18. Bratteby LE, Sandhagen B, Fan H, Samuelson G. A 7-day activity diary for assessment of daily energy expenditure validated by the doubly labeled water method in adolescents. Eur J Clin Nutr. 1997; 51: 585-91.

19. Cale L. Self-report measures of children's physical activity: recommendations for future development and a new alternative measure. Health Educ J. 1994; 53: 439-53.

20. Anção MS, Cuppari L, Tudisco LS, et al. Sistema de Apoio à Decisão em Nutrição - Versão 2.5. Centro de Informática em Saúde da Universidade Federal de São Paulo. Escola Paulista de Medicina. São Paulo; 1993.

21. American Academy of Pediatrics Committee on Nutrition. Statement on Cholesterol Pediatrics. 1992: 90: 469-73.

22. Casanueva VE, Cid XC, Calvo CM, et al. Lipoproteína en niños y adolescentes: relación con historia familiar de enfermedad coronaria. Rev Med Chile. 1996; 124: 799-804.

23. Kitange HM, Swai ABM, Masuki G, et al. Coronary heart disease risk factors in sub-Saharan Africa: studies in Tanzanian adolescents. J Epidemiol Community Health. 1993; 47: 303-7.

24. Andresen LB, Henckel P, Saltin B. Risk factors for cardiovascular disease in 16-19-year-old teenagers. J Int Med. 1989; 225: 157-63.

25. Webber LS, Harsha DW, Phillips GT, et al. Cardiovascular risk factors in Hispanic, white, and black children: The Brooks County and Bogalusa Heart Studies. Am J Epidemiol. 1991; 133: 704-14.

26. Lopez GR, Gonzales C, Castro JS, et al. Concentración de inulina y lípidos séricos en adolescentes de preparatoria en Guadalajara, México. Salud Pública de México. 2003; 45 Supl 1: S103-7.

27. Bergström E, Hernell O, La P, Vessby P. Serum lipid values in adolescents are related to family history, infant feeding, and growth. Atherosclerosis. 1995; 117: 1-13.

28. Porkka KVR, Viikari JSA, Rönnemmaa T, et al. Age and gender specific serum lipid and apolipoprotein fractiles of Finnish children and young adults. The Cardiovascular Risk in Young Finns Study. Acta Paediatr. 1994; 83: 838-48.

29. Paulus D, Saint-Remy A, Jean M. Smoking during adolescence: association with other cardiovascular risk factors in Belgian adolescents. Eur J Public Health. 2000; 10: 39-44.

30. Cohen B, Evers S, Manske Set al. Smoking, physical activity and breakfast consumption among secondary school students in a southwestern Ontario community. Can J Public Health. 2003; 94: 41-4.
31. O'Loughlin JL, Tarasuk J. Smoking, physical activity, and diet in North American Youth. Can J Public Health. 2003; 94: 27-30.

32. Kwiterovich PO. Plasma lipid and lipoprotein livels in childhood. Ann N Y Acad Sci. 1993; 623: 90-107.

33. Stozicky F, Slaby P, Volenikov L. Longitudinal study of serum cholesterol, apolipoproteins and sex hormones during puberty. Acta Paediatr Scand. 1991; 80: 1139-44.

34. Pedridou E, Malamou H, Doxiadis S, et al. Blood lipids in Greek adolescents and their relation to diet, obesity, and socioeconomic factors. Ann Epidemiol. 1995; 5: 286-91.

35. Katzmarzyk PT, Malina RM, Bouchard C. Physical activity, physical fitness, and coronary heart disease risk factors in youth: The Québec Family Study. Prev Med. 1999; 29: 555-62.

36. Tell GS, Vellar OD. Physical fitness, physical activity, and cardiovascular disease risk factors in adolescents: The Oslo Youth Study. Prev Med. 1988; $17: 12-24$

37. Paterno CA. Factores de riesgo coronario en la adolescencia. Estudo FRICELA. Rev Esp Cardiol. 2003; 56: 452-8.

38. Chu NF, Rimm EB, Wang DJ, et al. Clustering of cardiovascular disease risk factors among obese schoolchildren: The Taipei Children Heart Study. Am J Clin Nutr. 1998; 67: 1141-6.

39. Schmidt GJ, Walkuski JJ, Stensel DJ. The Singapore Youth Coronary Risk and Physical Activity Study. Med Sci Sports Exerc. 1998; 30: 105-13.

40. Lopez RE, Villa-Elizaga I, Goñi JS, et al. Estudio de Navarra (PECNA). Hiperlipidemias. Valores médios y percentilados de lípidos y lipoproteínas em uma población infanto-juvenil. Correlación com parâmetros antropométricos. An Esp Pediatr. 1993; 38: 307-15.

41. Romaldini $\mathrm{CC}$, Issler $\mathrm{H}$, Cardoso $\mathrm{AL}$, et al. Fatores de risco para aterosclerose em crianças e adolescentes com história familiar de doença arterial coronariana prematura. J Pediatr. 2004; 80: 13540.

42. Gerber ZRS, Zielinsky P. Fatores de risco de aterosclerose na infância: um estudo epidemiológico. Arq Bras Cardiol. 1997; 69: 231-6.

43. Forti N, Gionnini SD, Diament J, et al. Fatores de risco para doença arterial coronariana em crianças e adolescentes filhos de coronariopatas jovens. Arq Bras Cardiol. 1996; 66: 119-23.

44. Moura EC, Castro CM, Mellin AS, Figueiredo DB. Perfil lipídico em escolares de Campinas, SP, Brasil. Rev Saúde Pública. 2000; 34 : 499-505.

45. Neutzling MB, Taddei JAAC, Rodrigues EM, Sigulem DM. Overweight and obesity in Brazilian adolescents. Int J Obes. 2000; 24: 869-74.

46. Abrantes MM, Lamounier JA, Colosimo EA. Prevalência de sobrepeso e obesidade em crianças e adolescentes das regiões sudeste e nordeste. J Pediatr. 2002; 78: 335-40.

47. Gortmaker SL, Dietz WH, Sobol AM, Wehler CA. Increasing pediatric obesity in the United States. AJDC. 1987; 141: 535-40

48. Raitakari OT, Porkka KVK, Viikari JSA, et al. Clustering of risk factors for coronary heart disease in children and adolescents. The Cardiovascular Risk in Young Finns Study. Acta Paediatr. 1994; 83: 935-40.

49. Webber LS, Srinivasan SR, Wattigney WA, Berenson G. Tracking of serum lipids and lipoproteins from childhood to adulthood. The Bogalusa Heart Study. Am J Epidemiol. 1991; 133: 884-99.

50. Koch VH. Pressão arterial em pediatria. Aspectos metodológicos e normatização. Rev Bras Hipertens. 2000; 1: 71-8.

51. Bao W, Threefoot AS, Srinivasan SR, Berenson GS. Essential hypertension predicted by tracking of elevated blood pressure from childhood: The Bogalusa Heart Study. Am J Hypertens. 1995; 8: 657-65. 
52. Pennock LW, Fixler DE. Left ventricular hypertrophy in adolescents with elevated blood pressure: assessment by chest roentgenography, electrocardiography, echocardiography. Pediatrics. 1981; 67: 255-9.

53. Oliveira RG, Lamounier JÁ, Oliveira ADB, et al. Pressão arterial em escolares e adolescentes: Estudo Belo Horizonte. J Pediatr. 1999; 75: 256-66.

54. Brandão AP, Ferreira JO, Brandão AA, et al. Avaliação da pressão arterial em crianças e adolescentes: Estudo do Rio de Janeiro. HiperAtivo. 1996; 3: 86-92.

55. Berenson GS, Wattigney WA, Tracy RE. Atherosclerosis of the aorta and coronary arteries and cardiovascular risk factors in persons aged 6 to 30 years and studied at necropsy: the Bogalusa Heart Study. Am J Cardiol. 1992; 70: 851-8.

56. Freedman DS, Wattigney WA, Scrinivasan SR. The relation of atherosclerotic lesions to antemortem and postmortem lipid levels: the Bogalusa Heart Study. Atherosclerosis. 1993; 104: 37-46.

57. Andersen LB, Wedderkopp N, Hansen HS et al. Biological cardiovascular risk factors cluster in Danish children and adolescents: The European Youth Heart Study. Prev Med. 2003; 37: 363-7.

58. Maud EC, Bonetti LG. Prevalência de tabagismo e seus determinantes em algumas escolas de Barretos, São Paulo, em 1996. Rev Bras Cancerologia. 1999; 45: 41-4.

59. Bordin N, Nipper VB, Silva JO, Bortolomiol L. Prevalência de tabagismo entre escolares em municípios de área metropolitana da região Sul, Brasil, 1991. Cad Saúde Pública. 1993; 9: 185-9.

60. Ivanovic D, Castro $C$, Ivanovic $R$. Factores que inciden em el hábito de fumar de escolares de educación básica y media de Chile. Rev Saúde Pública. 1997; 31: 30-43.

61. Raitakari OT, Leino M, Raikkonen K. Clustering of risk habits in Young adults: The Cardiovascular Risk in Young Finns Study. Am J Epidemiol.
1995; 142: 36-44.

62. Jessor R. Risk behaviour in adolescent: a psychosocial frame-work for understanding and action. J Adolesc Health. 1991; 12: 597-605.

63. Woodward M, Bolton-Smith C, Tunstall-Pedoe H. Deficient health knowlwdge, diet and other lifestyles in smokers: Is a multifactorial approach required? Prev Med. 1994; 23: 354-61.

64. Twisk JWR, van Mechelen W, Kemper HCG. The relationship between lifestyle during adolescence and cardiovascular disease risk factors at adult age. The Amsterdam Growth and Health Longitudinal Study. Int J Sports Med. 2002; 23 Suppl 1: S8-S14.

65. Takada H, Harrell J, Deng S. Eating habits, activity, lipids and body mass index in Japanese children: The Shiratori Children Study. Int J Obes Relat Metab Disord. 1998; 22: 470-6.

66. Sallis JF; Owen N. Physical Activity and Behavioural Medicine. London: Sage; 1999.

67. Grundy SM, Denke MA. Dietary influences on serum lipids and lipoproteins. J Lipid Res. 1990; 31: 1149-72.

68. Guedes DP, Guedes JERP. Atividade física, aptidão cardiorrespiratória, composição da dieta e fatores de risco predisponentes às doenças cardiovasculares. Arq Bras Cardiol. 2001; 77: 243-50.

69. Craig WY, Palomaki GE, Johnson M, Haddow JE. Cigarette smokingassociated changes in blood lipid and lipoprotein levels in the 8-to-19year-old age group: a meta-analysis. Pediatrics. 1990; 85: 155-8.

70. Bayle MS, Requejo AG, Ruiz-Jarabo C, et al. Smoking and apolipoproteins in adolescents. J Adolesc Health. 1992; 13: 524-7.

71. Price JF, Mowbray PI, Lee AJ, et al. Relationship between smoking and cardiovascular risk factors in the development of peripheral arterial disease and coronary artery disease. Edinburgh Artery Study. Eur Heart J. $1999 ; 20: 344-53$ 